\title{
SOME NON-CRITICAL IDEMPOTENTS IN THE CLOSURE OF THE CHARACTERS IN THE MAXIMAL IDEAL SPACE OF $M\left(D_{2}\right)$
}

\author{
JANE LAKE \\ (Received 3 April 1981; revised 29 September 1981) \\ Communicated by W. Moran
}

\begin{abstract}
This paper shows that the idempotent generalized characters associated with a Raikov System generated by a $K_{2}$ set in $\mathbf{D}_{2}=\prod_{i=1}^{\infty}\left(\mathbf{Z}_{2}\right)_{i}$ is contained in the closure of the characters $\overline{\mathbf{D}_{2}}$ in $\Delta M\left(\mathbf{D}_{2}\right)$. 1980 Mathematics subject classification (Amer. Math. Soc.): 43 A 46.
\end{abstract}

\section{Introduction}

We will be working with the compact totally disconnected abelian group $\mathbf{D}_{2}=$ $\Pi_{i=1}^{\infty}\left(\mathbf{Z}_{2}\right)_{i}$ which has dual group $\hat{\mathbf{D}_{2}}=\bigoplus_{i=1}^{\infty}\left(\mathbf{Z}_{2}\right)_{i}$ where $\mathbf{Z}_{2}$ is the multiplicative group of order $2, \mathbf{Z}_{2}=\{1,-1 ; \cdot\}$. The dual group $\hat{\mathbf{D}_{2}}$ is canonically embedded in $\Delta M\left(\mathrm{D}_{2}\right)$, the maximal ideal space of $M\left(\mathrm{D}_{2}\right)$.

A compact perfect subset $K$ of $\mathbf{D}_{2}$ is called a $K_{2}$ subset of $\mathbf{D}_{2}$ if for any continuous function $f: K \rightarrow \overrightarrow{Z_{2}}$ there is a character $\phi \in \hat{\mathbf{D}_{2}}$ such that $\phi$ restricted to $K$ is equal to $f$.

It will be shown that the idempotent associated with any Raikov System generated by a $K_{2}$ set is contained in the closure of the characters $\overline{\mathbf{D}_{2}}$ in $\Delta M\left(\mathbf{D}_{2}\right)$. Dunkl and Ramirez (1972) have shown that the idempotent associated with the Raikov System generated by a closed subgroup is in the closure of the characters.

As the maximal ideal space $\Delta M\left(\mathrm{D}_{2}\right)$ has the weak topology from the FourierStieltjes transforms of the measures in $M\left(\mathbf{D}_{2}\right)$, an idempotent associated with a Raikov System is in the closure of the characters if and only if the Fourier-Stieltjes

(C) Copyright Australian Mathematical Society 1983 
transforms of the measures satisfy the following condition:

For all measures $\mu$ concentrated on the Raikov System and for all measures $\nu$ which annihilate all the sets in the Raikov System

$$
\|\hat{\mu}\|_{\infty} \leqslant\left\|(\mu+\nu)^{\hat{n}}\right\|_{\infty}
$$

where the sup norm is taken over $\hat{\mathbf{D}_{2}}$.

We will prove that Raikov Systems generated by $K_{2}$ subsets of $\mathbf{D}_{2}$ satisfy this bound by constructing a series of positive definite functions such that, for each measure $\mu$ and $\nu$ as above, there is a positive definite function $P_{\gamma}$ such that

$$
\left|\int_{\mathbf{D}_{2}} P_{\gamma} d \mu-\hat{\mu}(1)\right|<\varepsilon \text { and }\left|\int_{\mathbf{D}_{2}} P_{\gamma} d \nu\right|<\varepsilon .
$$

\section{Acknowledgement}

The following research was done while the author was working towards a Ph.D. at the University of New South Wales, supported by a Commonwealth Postgraduate Research Award. The author wishes to express her thanks to her supervisor Gavin Brown for his help and encouragement.

\section{Raikov systems and idempotents}

Let $G$ be a locally abelian group and let $A$ be a subset of $G$. We define the Raikov System of sets of $G$ generated by $A, \Re_{A}$, to be the collection of all measurable subsets of some countable union of translates of sums of $A$.

That is to say

$$
\mathscr{R}_{A}=\left\{\begin{array}{c}
1 . B \text { is measurable; } \\
B \subseteq G: 2 . \exists x_{i} \in G, m_{i} \in \mathbf{Z}^{+}, \text {for } i \in \mathbf{Z}^{+}, \infty \\
\text { such that } B \subseteq \bigcup_{i=1}^{\infty} x_{i}+\left(m_{i}\right) A
\end{array}\right\}
$$

where for $m \in \mathbf{Z}^{+}$,

$$
\begin{gathered}
(m) A=A+A+\cdots+A=\left\{\sum_{i=1}^{m} x_{i}: x_{i} \in A, i=1, \ldots, m\right\} . \\
m \text { times }
\end{gathered}
$$

We notice that $\Re_{A}$ is closed under translation, intersection, countable unions and addition of sets.

The Raikov System $\Re_{A}$ is now used to define a direct sum splitting of $M(G)$ into the $L$-algebra $\mathbb{Q}_{A}$ of measures concentrated on the sets in the Raikov System, and the $L$-ideal $\Phi_{A}$ of measures which annihilate all the sets in the Raikov System. 
That is to say,

$$
\begin{aligned}
& \mathscr{Q}_{A}=\left\{\mu \in M(G): \exists B \in \Re_{A} \text { such that } \mu \text { is concentrated on } B\right\}, \text { and } \\
& \mathscr{\Phi}_{A}=\left\{\nu \in M(G):|\nu|(B)=0 \text { for all } B \in \Re_{A}\right\} .
\end{aligned}
$$

The idempotent $I_{A}$ associated with this Raikov system is the projection from $M(G)$ onto $\mathbb{Q}_{A}$ and hence is a homomorphism and an element of the maximal ideal space of $M(G)$.

The group of characters on $G, \hat{G,}$ is canonically embedded in $\Delta M(G)$ and the idempotent $I_{A}$ is contained in the closure of the characters $\bar{G}$ in $\Delta M(G)$ if and only if the projection

$$
I_{A}: M(G) \rightarrow \mathbb{Q}_{A}
$$

is bounded in the Fourier-Stieltjes transform norm, that is to say, for any measure $\mu \in M(G)$

$$
\left\|\left(I_{A} \mu\right)^{\hat{n}}\right\|_{\infty} \leqslant\|\hat{\mu}\|_{\infty}
$$

where the sup norm is taken over $\hat{G}$. If we have two direct sum splittings of $M(G)$ into an $L$-subalgebra and $L$-ideal associated with idempotents in the closure of the characters, then the direct sum splitting

$$
\mathbb{Q}_{1} \cap \mathbb{Q}_{2} \oplus \mathscr{G}_{1}+\mathscr{G}_{2}
$$

is also a splitting of $M(G)$ associated with an idempotent in the closure of the characters of $G$.

\section{Properties of $K_{2}$ sets}

Let $K \subseteq \mathrm{D}_{2}$ be a compact perfect $K_{2}$ subset of $\mathrm{D}_{2} . \overline{\mathrm{Gp} K}$ is a closed subgroup of $\mathrm{D}_{2}$ and so $\mathrm{D}_{2}=\overline{\mathrm{Gp} K} \oplus H$ where $H$ is also a closed subgroup of $\mathrm{D}_{2} . \overline{\mathrm{Gp} K}$ is isomorphic to $\mathbf{D}_{2}$ and the idempotent associated with the Raikov System generated by $\overline{\mathrm{Gp} K}$ is in the closure of the characters (Dunkl and Ramirez (1972)).

If we have a Raikov splitting of $M\left(\mathrm{D}_{2}\right)$ generated by a set $A \subseteq \overline{\mathrm{Gp} K}$ then the idempotent associated with this splitting is in the closure of the characters if and only if the condition

$$
\left\|\left(I_{A} \mu\right)^{\hat{n}}\right\|_{\infty} \leqslant\|\hat{\mu}\|_{\infty}
$$

holds for all measures $\nu$ in $M(\overline{\mathrm{Gp} K})$. For convenience therefore we assume that $\overline{\mathrm{Gp} K}=\mathrm{D}_{2}$.

For any continuous function $\phi: K \rightarrow \overrightarrow{\mathbf{Z}_{2}}$ there is a character $\chi \in \hat{\mathbf{D}_{2}}$ such that $\left.\chi\right|_{K}=\phi$. For each character $\chi$ in $\hat{\mathbf{D}_{2}}$ we let $P_{\chi}=\{x \in K: \chi(x)=-1\}$. We say 
that a set of characters $\left\{\chi_{1} \cdots \chi_{n}\right\}$ determines a partition of $K$ if

1 .

$$
\begin{gathered}
P_{\chi_{i}} \cap P_{\chi_{j}}=\phi \quad \forall i \neq j, 1 \leqslant i, j \leqslant n ; \\
K=\bigcup_{i=1}^{n} P_{\chi_{i}} .
\end{gathered}
$$

Given two partitions $\mathscr{P}=\left\{P_{\chi_{1}}, P_{\chi_{2}}, \ldots, P_{\chi_{n}}\right\}$ and $\mathscr{P}^{\prime}=\left\{P_{\phi_{1}}, P_{\phi_{2}}, \ldots, P_{\phi_{m}}\right\}$ of $K$ determined by the characters $\left\{\chi_{1}, \chi_{2}, \ldots, \chi_{n}\right\}$ and $\left\{\phi_{1}, \ldots, \phi_{m}\right\}$ respectively we say $\mathscr{P}$ is an everywhere finer partition of $K$ than $\mathcal{P P}^{\prime}$ if for each $1 \leqslant i \leqslant m$ there exists an $I_{i} \subseteq\{1, \ldots, n\}$ with $\# I_{i} \geqslant 2$ such that

$$
P_{\phi_{i}}=\bigcup_{j \in I_{i}} P_{\chi_{j}} .
$$

Since $\overline{\mathrm{Gp} K}=\mathbf{D}_{2}$ this implies that

$$
\phi_{i}=\prod_{j \in I_{i}} \chi_{j} .
$$

As $K$ is a $K_{2}$ subset of $\mathbf{D}_{2}$, for any continuous function $f$ from $K$ into $Z_{2}$ there is a partition (in fact, trivial) $\mathscr{P}=\left\{P_{\chi_{1}}, P_{\chi_{2}}, \ldots, P_{\chi_{m}}\right\}$ such that

$$
f=\left.\prod_{j \in I} \chi_{j}\right|_{K} \text { for some } I \subseteq\{1,2, \ldots, m\} .
$$

We say that the function $f$ can be generated by the partition $\mathscr{P}$.

Given two partitions of $K, \mathscr{P}=\left\{P_{\phi_{1}}, P_{\phi_{2}}, \ldots, P_{\phi_{n}}\right\}$ and $\mathscr{P}^{\prime}=\left\{P_{\chi_{1}}, P_{x_{2}}, \ldots, P_{x_{m}}\right\}$, there exists a partition $\mathscr{P}^{\prime \prime \prime}$ which is everywhere finer than $\mathscr{P}$ and $\mathcal{P}^{\prime}$ since, for each $1 \leqslant i \leqslant m$ and $1 \leqslant j \leqslant n$, if we have that $P_{\chi_{1}} \cap P_{\phi_{j}} \neq \varnothing$ then there exists a character $\omega_{i j}$ such that

$$
\omega_{i j}= \begin{cases}-1 & \text { on } P_{\chi_{i}} \cap P_{\phi_{j}} \\ 1 & \text { elsewhere on } K\end{cases}
$$

and so the partition determined by the characters

$$
\left\{\omega_{i j}: P_{\chi_{i}} \cap P_{\phi_{j}} \neq \varnothing, 1 \leqslant i \leqslant m, i \leqslant j \leqslant n\right\}
$$

is finer than $\mathcal{P}$ and $\mathscr{P}^{\prime}$. Since $K$ is totally disconnected there exists a partition $\mathscr{P}^{\prime \prime \prime}$ which is everywhere finer than $\left\{\omega_{i j}: P_{\chi_{i}} \cap P_{\phi_{j}} \neq \varnothing, 1 \leqslant i \leqslant m, 1 \leqslant j \leqslant n\right\}$ and so is everywhere finer than both $\mathscr{P}$ and $\mathcal{P}^{\prime}$.

We define a sequence of partitions of $K,\left\{\mathscr{P}_{i}\right\}_{i \in \mathbf{Z}^{+}}$where

$$
\mathscr{P}_{i}=\left\{P_{\phi_{1}^{\prime}}, P_{\phi_{2}^{\prime}}, \ldots, P_{\phi_{j(i)}^{\prime}}\right\}
$$

determined by a set of characters $\left\{\phi_{1}^{i}, \phi_{2}^{i}, \ldots, \phi_{j(i)}^{i}\right\}$, to be a "separating sequence of partitions of $K$ " if

1. $\forall n \in \mathbf{Z}^{+} \mathscr{P}_{n+1}$ is an everywhere finer partition of $K$ than $\mathscr{P}_{n}$;

2. for each continuous function $f: K \rightarrow \mathbf{Z}_{2}$ there is an $N \in \mathbf{Z}^{+}$such that $f$ can 
be generated by the partition $\mathscr{P}_{N}$ and hence $f$ can be generated by each partition $\mathscr{P}_{n}$ for $n \geqslant N$.

LEMMA 1. Let $K$ be a $K_{2}$ subset of $\mathbf{D}_{2}$. Then there is a separating sequence of partitions of $K$.

Proof. The set of continuous functions from $K$ into $\mathbf{Z}_{2}$ is countable: denote it by $\left\{f_{i}: i \in \mathbf{Z}^{+}\right\}$. We will define the sequence of partitions inductively. Let $\mathscr{P}_{1}$ be a partition of $K$ which generates $f_{1}$. Let $\mathscr{P}_{2}$ be a partition of $K$ everywhere finer than $\mathscr{P}_{1}$ which also generates $f_{2}$. Inductively, let $\mathscr{P}_{n+1}$ be an everywhere finer partition than $\mathscr{P}_{n}$ that generates $f_{n+1}$ and hence also generates $f_{1}, f_{2}, \ldots, f_{n}$.

We can now characterize elements of $K,(m) K$ and $\overline{\mathrm{Gp} K}$ using a separating sequence of partitions of $K$. Let $\left\{\mathscr{P}_{n}\right\}_{n \in \mathbf{Z}^{+}}$, where $\mathscr{P}_{n}=\left\{P_{\phi_{1}^{n}}, P_{\phi_{2}^{n}}, \ldots, P_{\phi_{j(n)}^{n}}\right\}$, be a separating sequence of partitions of $K$. We define a sequence of characters $\left\{\phi_{k(m)}^{m}\right\}_{m \in \mathbf{Z}^{+}}$where $1 \leqslant k(m) \leqslant j(m)$ to be a "chain" of characters from the separating sequence $\left\{\mathscr{P}_{n}\right\}_{n \in \mathbf{Z}^{+}}$if

$$
P_{\phi_{k(1)}^{1}} \supset P_{\phi_{k(2)}^{2}} \supset P_{\phi_{k(3)}^{3}} \supset \cdots \supset P_{\phi_{k(n)}^{n}} \supset P_{\phi_{k(n+1)}^{n+1}} \supset \cdots .
$$

Obviously if we have two chains $\left\{\phi_{k(i)}^{i}\right\}_{i \in \mathbf{Z}^{+}}$and $\left\{\phi_{m(i)}^{i}\right\}_{i \in \mathbf{Z}^{+}}$such that for some $N \in \mathbf{Z}^{+}, \phi_{k(N)}^{N} \neq \phi_{m(N)}^{N}$, then $\phi_{k(n)}^{n} \neq \phi_{m(n)}^{n}$ for all $n \geqslant N$. We also have the following lemma.

LEMMA 2. Given $K$ a $K_{2}$ subset of $\mathbf{D}_{2}$ with $\overline{\mathrm{Gp} K}=\mathbf{D}_{2}$, and $\left\{\mathscr{P}_{i}\right\}_{i \in \mathbf{Z}^{+}}$a separating sequence of partitions of $K$, then $K$ is equal to the set $H$ where

$$
H=\left\{x \in \mathbf{C}_{2}: \begin{array}{l}
\exists \text { chain }\left\{\phi_{k(i)}^{i}\right\}_{i \in \mathbf{Z}^{+}} \text {of characters from the separating } \\
1 . \phi_{k(i)}^{i}(x)=-1 \quad \forall i \in \mathbf{Z}^{+} \\
\text {2. } \phi_{m}^{i}(x)=1 \quad \forall m \neq k(i), 1 \leqslant m \leqslant j(i) .
\end{array}\right\}
$$

Proof. Obviously $K \subseteq H$. Let $\left\{\phi_{k(i)}\right\}_{i \in \mathbf{Z}^{+}}$be a chain of characters from the separating sequence of $K$. By definition $P_{\phi_{k(i)}^{i}} \subseteq K$, and so we have $\cap_{i \in \mathbf{Z}^{+}} P_{\phi_{k(i)}^{i}}$ is non empty, as $\left\{\phi_{k(i)}^{i}\right\}_{i \in \mathbf{Z}^{+}}$is a chain, and so $\bigcap_{i \in \mathbf{Z}^{+}} P_{\phi_{k(i)}^{i}}=\{x\}$ for some $x \in K$ as $\left\{\mathscr{P}_{i}\right\}_{i \in \mathbf{Z}^{+}}$is a separating sequence of partitions of $K$. So we have

$$
\phi_{k(i)}^{i}(x)=-1 \quad \forall i \in \mathbf{Z}^{+}
$$

and

$$
\phi_{m}^{i}(x)=1, \quad m \neq k(i), \forall 1 \leqslant m \leqslant j(i) .
$$


To show that $K=H$, we need to show that for each chain $\left\{\phi_{k(i)}^{i}\right\}_{i \in \mathbf{Z}^{+}}$from the separating sequence there is a unique $x \in \mathbf{D}_{2}$ with

$$
\phi_{k(i)}^{i}(x)=-1 \quad \forall i \in \mathbf{Z}^{+}
$$

and

$$
\phi_{m}^{i}(x)=1 \quad \forall m \neq k(i), 1 \leqslant m \leqslant j(i) .
$$

Assume $x, y \in \mathbf{D}_{2}$ with

$$
\phi_{k(i)}^{i}(x)=\phi_{k(i)}^{i}(y)=-1 \quad \forall i \in \mathbf{Z}^{+}
$$

and

$$
\phi_{m}^{i}(x)=\phi_{m}^{i}(y)=1 \quad \forall m \neq k(i), 1 \leqslant m \leqslant j(i),
$$

so if $x$ and $y$ are distinct there must exist a character $\chi \in \hat{\mathbf{D}_{2}}$ such that $\chi(x) \neq \chi(y)$, but $\overline{\mathrm{Gp} K}=\mathbf{D}_{2}$ so $\left.\chi\right|_{K}$ is not identically equal to 1 . As $\left\{\mathscr{P}_{i}\right\}_{i \in \mathbf{Z}^{+}}$is a separating sequence of partitions of $K$ there must be an $i \in \mathbf{Z}^{+}$such that $\chi$ is generated by the functions $\left\{\phi_{1}^{i}, \phi_{2}^{i}, \ldots, \phi_{j(i)}^{i}\right\}$ on $K$. Thus

$$
\phi_{m}^{i}(x) \neq \phi_{m}^{i}(y) \text { for some } 1 \leqslant m \leqslant j(i)
$$

and so $x=y$.

As $\overline{\mathrm{GpK}}=\mathrm{D}_{2}$, every character on $\mathrm{D}_{2}$ is uniquely determined by its restrictions to $K$, so given $\left\{\mathcal{P}_{i}\right\}_{i \in \mathbf{Z}^{+}}$, a separating sequence of partitions of $K$, we have that every element of $\mathbf{D}_{2}$ is uniquely determined by the values of the $\phi_{k}^{i}(x)$ where $\phi_{k}^{i}$ are the characters from the separating sequence $\left\{\mathscr{P}_{i}\right\}_{i \in \mathbf{Z}^{+}}$.

For $x \in \overline{\mathrm{Gp} K}=\mathbf{D}_{2}$ we define the length of $x$ on the $n$th partition of $K$ to be

$$
l(n, x)=\sum_{i=1}^{u(n)} \frac{1}{2}\left(1-\phi_{i}^{n}(x)\right)=\#\left\{\phi_{i}^{n}: \phi_{i}^{n}(x)=-1,1 \leqslant i \leqslant j(n)\right\} .
$$

We now have a lemma.

Lemma 3. Let $K$ be a $K_{2}$ subset of $\mathbf{D}_{2}$ with $\overline{\mathrm{Gp} K}=\mathbf{D}_{2}$ and let $\left\{\mathscr{P}_{n}\right\}_{n \in \mathbf{Z}^{+}}$be a separating sequence of partitions of $K$ with associated length function $l(n,$.$) . Then:$

1. For $x \in K, l(n, x)=1 \forall n \in \mathbf{Z}^{+}$.

2. For $x \in(m) K \backslash \bigcup_{i=1}^{m-1}(i) K, \lim _{n \rightarrow \infty} l(n, x)=m$.

3. For $x \in \overline{\mathrm{Gp} K}, n \in \mathbf{Z}^{+}$

a) if $l(n, x)=0$ then $l(m, x)=0 \forall m \leqslant n$,

b) $l(n+1, x) \geqslant l(n, x)$.

4. For $x \in \overline{\mathrm{Gp} K} \backslash \bigcup_{i=1}^{\infty}(i) K, \lim _{n \rightarrow \infty} l(n, x)=\infty$. 
Proof. 1. We can see from Lemma 2 that each $x \in K$ is uniquely associated with a chain of characters, say $\left\{\phi_{k(i)}^{i}\right\}_{i \in \mathbf{Z}^{+}}$, from the separating sequence with

$$
\phi_{k(i)}^{i}(x)=-1 \quad \forall i \in \mathbf{Z}^{+}
$$

and

$$
\phi_{m}^{i}(x)=1 \quad \forall 1 \leqslant m \leqslant j(i), m \neq k(i),
$$

and so $l(n, x)=1$.

2. $x=x_{1}+x_{2}+\cdots+x_{m}, x_{i} \in K$, all distinct. Each $x_{i}$ is uniquely associated with a chain $\left\{\phi_{k(n, i)}^{n}\right\}_{n \in \mathbf{Z}^{+}}$from the separating sequence with

$$
\phi_{k(n, i)}^{n}\left(x_{i}\right)=-1 \quad \forall n \in \mathbf{Z}^{+}
$$

and

$$
\phi_{j}^{n}\left(x_{i}\right)=1, \quad 1 \leqslant j \leqslant j(n), j \neq k(n, i) .
$$

As the $x_{i}$ are distinct there exists an $N \in \mathbf{Z}^{+}$with

$$
\phi_{k(N, i)}^{N} \neq \phi_{k(N, j)}^{N} \quad \forall i \neq j, 1 \leqslant i, j \leqslant m
$$

and so

$$
\phi_{k(n, i)}^{n} \neq \phi_{k(n, j)}^{n} \quad \forall i \neq j, 1 \leqslant i, j \leqslant m \text { and } n \geqslant N
$$

so $l(n, x)=m$ for all $n \geqslant N$.

3. a) As $\overline{\mathrm{GpK}}=\mathbf{D}_{2}$ and $\left\{\mathscr{P}_{i}\right\}_{i \in \mathbf{Z}^{+}}$is a separating sequence of partitions of $K$, letting

$$
H_{n}=\left\{x \in \mathbf{D}_{2}: l(i, x)=0, i=1, \ldots, n\right\}
$$

we have that $\left\{H_{n}: n \in \mathbf{Z}^{+}\right\}$forms a base of open neighbourhoods of zero in $\mathbf{D}_{2}$. Let $x \in \overline{\mathrm{GpK}}=\mathbf{D}_{2}$ be such that $l(n, x)=0$. We can write

$$
x=x_{1}+x_{2}+\cdots+x_{r}+h
$$

where $h \in H_{n+1}$ and $x_{i} \in K, i=1, \ldots, r$ are distinct, so each $x_{i}$ is uniquely associated with a chain $\left\{\phi_{k(m, i)}^{m}\right\}_{m \in \mathbf{Z}^{+}}$where $\phi_{k(m, i)}^{m}\left(x_{i}\right)=-1$ and

$$
\phi_{j}^{m}\left(x_{i}\right)=1, \quad 1 \leqslant j \leqslant j(m), j \neq k(m, i) .
$$

Now $l(n, x)=l\left(n, x_{1}+x_{2}+\cdots+x_{r}\right)=0$ so we must be able to group the $x_{i}$ in pairs $x_{i}, x_{j}$ with $\phi_{k(n, i)}^{n}=\phi_{k(n, j)}^{n}$, and so

$$
\phi_{k(m, i)}^{m}=\phi_{k(m, j)}^{m} \quad \text { for all } m \leqslant n .
$$

Thus

$$
l\left(m, x_{1}+x_{2}+\cdots+x_{r}\right)=0 \quad \forall m \leqslant n
$$

so $l(m, x)=0$ for all $m \leqslant n$.

3. b) For $x \in \overline{\mathrm{Gp} K}=\mathbf{D}_{2}$ let $l(n+1, x)=m$. Then we can write $x=x_{1}+x_{2}$ $+\cdots+x_{m}+h$ where $l(n+1, h)=0$, so

$$
l(i, h)=0, \quad 1 \leqslant i \leqslant n+1,
$$


and so

$$
l(n, x)=l\left(n, x_{1}+x_{2}+\cdots+x_{m}\right) \leqslant m \leqslant l(n+1, x) .
$$

4. Let $x \in \overline{\operatorname{Gp} K}$ and suppose that $\lim _{n \rightarrow \infty} l(n, x)=m$, so for each $n \in \mathbf{Z}^{+}$we can find an $h \in H_{n}$ and $x_{1} \cdots x_{m} \in K$ so that

$$
x=x_{1}+x_{2}+\cdots+x_{m}+h .
$$

As $\left\{H_{n}: n \in \mathbf{Z}^{+}\right\}$forms a base of open neighbourhoods of zero we have that

$$
x \in \overline{(m) K}=(m) K \text {. }
$$

\section{Positive definite functions}

We will now use the separating sequence of partitions of $K,\left\{\mathscr{P}_{m}\right\}_{m \in \mathbf{z}^{+}}$, with generating characters $\left\{\phi_{1}^{m}, \phi_{2}^{m}, \ldots, \phi_{j(m)}^{m}\right\}$, for $m \in \mathbf{Z}^{+}$, to construct a sequence of positive definite functions on $\mathbf{D}_{2}$.

LEMMA. Let $r \in(0,1)$ and $n \in \mathbf{Z}^{+}$. Then the function

$$
\begin{aligned}
& F_{r}^{n}: \overline{\mathrm{Gp} K} \rightarrow \mathbf{R} \\
&: x \sim r^{/(n, x)}
\end{aligned}
$$

is a positive definite function on $\mathbf{D}_{2}=\overline{\mathrm{Gp} K}$.

ProOF. Consider the $n$th partition of $K, \mathscr{P}_{n}=\left\{P_{\phi_{1}^{n}}, P_{\phi_{2}^{n}}, \ldots, P_{\phi_{j(n)}^{n}}\right\}$, generated by the characters $\left\{\phi_{1}^{n}, \phi_{2}^{n}, \ldots, \phi_{j(n)}^{n}\right\}$. The measure $\mu_{n, r}$ on $\mathbf{D}_{2}$

$$
\mu_{n, r}=\underset{i=1}{j(n)}\left(\left(\frac{1+r}{2}\right) \delta(1)+\left(\frac{1-r}{2}\right) \delta\left(\phi_{i}^{n}\right)\right)
$$

is a positive measure for $r \in(0,1)$ and has Fourier transform

$$
\hat{\mu}_{n, r}(x)=r^{l(n, x)}
$$

and so $F_{r}^{n}: \mathbf{D}_{2} \rightarrow \mathbf{R}: x \sim r^{l(n, x)}$ is a positive definite function on $\mathbf{D}_{2}=\overline{\mathrm{Gp} K}$.

We now have the main theorem of this section.

TheOREM 1. Let $K \subseteq \mathbf{D}_{2}$ be a $K_{2}$ subset of $\mathbf{D}_{2}$ such that $\overline{\mathrm{Gp} K}=\mathbf{D}_{2}$. Then, for each $m \in \mathbf{Z}^{+}$and $\varepsilon, \delta>0$ we can choose an $h \in \mathbf{Z}^{+}$such that, for any open neighbourhood $H$ of zero, there exists a positive definite function $F$ with $F(0)=1$ 
and

$$
\begin{aligned}
& \text { 1. } F(x)>1-\varepsilon \text { for } x \in \bigcup_{i=1}^{m}(i) K, \\
& \text { 2. }|F(x)|<\delta \text { for } x \in \mathbf{D}_{2} \backslash\left\{\left(\bigcup_{j=1}^{m+h}(i) K\right)+H\right\} \text {. }
\end{aligned}
$$

Proof. Choose an $r \in(0,1)$ such that $\left(1-r^{m}\right)<\varepsilon$ and choose an $h \in \mathbf{Z}^{+}$so that $r^{m+h}<\delta$. Now let $H^{\prime}$ be an open neighbourhood of zero of the form

$$
H^{\prime}=\left\{x \in \mathbf{D}_{2}: \phi_{j}^{i}(x)=1 \forall 1 \leqslant j \leqslant j(i), 1 \leqslant i \leqslant I\right\}=H_{I}
$$

for some $I \in \mathbf{Z}^{+}$. So we have

$$
\begin{aligned}
\left\{\left(\bigcup_{i=1}^{m+h}(i) K\right)+H^{\prime}\right\} & =\left\{x \in \mathbf{D}_{2}: l(p, x) \leqslant m+h \text { for } 1 \leqslant p \leqslant I\right\} \\
& =\left\{x \in \mathbf{D}_{2}: l(I, x) \leqslant m+h\right\} .
\end{aligned}
$$

Now observe that $F_{r}^{I}(x)=r^{l(I, x)}$; so, for $x \in \cup_{i=1}^{m}(i) K$,

$$
\left|1-F_{r}^{I}(x)\right| \leqslant\left|1-r^{m}\right|<\varepsilon .
$$

For $x \in \mathbf{D}_{2} \backslash\left\{\left(\cup_{i=1}^{m+h}(i) K\right)+H^{\prime}\right\}$ we have $l(I, x)>m+h$, so $F_{r}^{I}(x) \leqslant$ $r^{m+h}<\delta$.

5.

We now prove a general theorem about Raikov idempotent generalized characters in the closure of the characters of $\mathbf{D}_{2}$, given the existence of positive definite functions with certain properties. The main result is then a corollary of Theorem 1 and the following theorem.

THEOREM 2. Let $A \subseteq \mathbf{D}_{2}$ be a compact perfect subset of $\mathbf{D}_{2}$ such that, for every $m \in \mathbf{Z}^{+}, \varepsilon, \delta>0$ and open neighbourhood $H$ of zero, there exists an integer $h \in \mathbf{Z}^{+}$independent of the neighbourhood $\mathrm{H}$ and a positive definite function $\mathrm{F}$ on $\mathbf{D}_{2}$ with

1. $F(0)=0$;

2. $|F(x)-1|<\varepsilon \quad \forall x \in \bigcup_{i=1}^{m}(i) A$;

3. $|F(x)|<\delta \quad \forall x \in \mathbf{D}_{2} \backslash\left\{\left(\bigcup_{i=1}^{m+h}(i) A\right)+H\right\}$. 
Then the idempotent generalized character $I_{A}$ associated with the Raikov System generated by $A$ on $\mathbf{D}_{2}$ is in the closure of the characters $\overline{\mathbf{D}_{2}}$ in $\Delta M\left(\mathbf{D}_{2}\right)$.

Proof. $\Delta M\left(\mathrm{D}_{2}\right)$ has the weak topology induced from the Fourier-Stieltjes transforms of the measures in $M\left(\mathbf{D}_{2}\right)$, so the idempotent $I_{A}$ is in $\overline{\mathbf{D}_{2}}$ if and only if

$$
\left\|\left(I_{A} \mu\right) \hat{\|}\right\|_{\infty} \leqslant\|\hat{\mu}\|_{\infty} \quad \forall \mu \in M\left(\mathbf{D}_{2}\right)
$$

where the sup norms are taken over $\hat{\mathbf{D}_{2}}$.

Let $\mu \in \mathbb{Q}_{A}$ and $\varepsilon>0$. We can find an $l \in \mathbf{Z}^{+}$so that

$$
\mu=\sum_{i=1}^{n} \delta_{x_{i}} * \mu_{i}+\mu^{\prime}
$$

where $\mu_{i} \in M\left(\bigcup_{j=1}^{l}(j) A\right),\|\mu\|<\varepsilon$ and $x_{i} \in \mathbf{D}_{2}$. We will consider the measure $\sum_{i=1}^{n} \delta_{x_{i}} * \mu_{i}$ which is concentrated on

$$
\bigcup_{j=1}^{n}\left(x_{j}+\bigcup_{i=1}^{l}(i) A\right) \text {. }
$$

We can assume (without loss of generality) that $S=\left\{x_{1}, x_{2}, \ldots, x_{n}\right\}$ is a finite subgroup of $\mathrm{D}_{2}$. We can find a subgroup $S_{0}$ of $S$ such that

$$
S+\mathrm{Gp} A=S_{0}+\mathrm{Gp} A \text { and } S_{0} \cap \mathrm{Gp} A=\{0\}
$$

and can find an $m \in \mathbf{Z}^{+}$so that

$$
\bigcup_{x \in S}\left(x+\bigcup_{i=1}^{l}(i) A\right) \subset \bigcup_{y \in S_{0}}\left(y+\bigcup_{i=1}^{m}(i) A\right) .
$$

Now we have for each $q \in \mathbf{Z}^{+}$and $x \neq y \in S_{0}$ that

$$
\left\{x+\bigcup_{i=1}^{q}(i) A\right\} \cap\left\{y+\bigcup_{i=1}^{q}(i) A\right\}=\varnothing
$$

so we can choose an open neighbourhood $H(q)$ of zero such that for all $x, y \in S_{0}, x \neq y$,

$$
\left\{\left(x+\bigcup_{i=1}^{q}(i) A\right)+H(q)\right\} \cap\left\{\left(y+\bigcup_{i=1}^{q}(i) A\right)+H(q)\right\}=\varnothing .
$$

Now choose an $h \in \mathbf{Z}^{+}$such that for every open neighbourhood $H$ of zero there exists a positive definite function $F$ on $\mathrm{D}_{2}$ with

1. $F(0)=1$;

2. $|F(x)-1|<\varepsilon \quad \forall x \in \bigcup_{i=1}^{m}(i) A$;

3. $|F(x)|<\frac{\varepsilon}{\left|S_{0}\right|} \quad \forall x \in \mathbf{D}_{2} \backslash\left\{\left(\bigcup_{i=1}^{m+h}(i) A\right)+H\right\}$. 
Let $H$ be an open neighbourhood of zero contained in $H(m+h)$. Then we can find a positive definite function $F$ satisfying

$$
\begin{array}{ll}
\text { 1. } & F(0)=1 ; \\
\text { 2. }|F(x)-1|<\varepsilon & \forall x \in \bigcup_{i=1}^{m}(i) A ; \\
\text { 3. }|F(x)|<\frac{\varepsilon}{\left|S_{0}\right|} \quad \forall x \in \mathbf{D}_{2} \backslash\left\{\left(\bigcup_{i=1}^{m+h}(i) A\right)+H\right\} .
\end{array}
$$

Now the measure $\left|S_{0}\right| \cdot m_{S_{0}}$ where $m_{S_{0}}$ is haar measure on $S_{0}$ has positive Fourier transform, so $\mathscr{F}=\left|S_{0}\right| \cdot m_{S_{0}} * F$ is a positive definite function on $\mathbf{D}_{2}$, and

$$
\mathscr{F}=\sum_{x \in S_{0}} \delta_{x} * F
$$

$\mathscr{F}$ has the following properties:

1. $\mathscr{F}(0) \leqslant 1+\varepsilon$.

2. $|\mathscr{F}(x)-1|<2 \varepsilon, \quad x \in \bigcup_{y \in S_{0}}\left(y+\bigcup_{i=1}^{m}(i) A\right)$.

3. $|\mathcal{F}(x)|<\varepsilon, \quad x \in \mathbf{D}_{2} \backslash\left\{\left(\bigcup_{y \in S_{0}} y+\bigcup_{i=1}^{m+h}(i) A\right)+H\right\}$.

Let $\nu$ be a measure in $I_{A}$. Then

$$
|\nu|\left(\bigcup_{y \in S_{0}}\left(y+\bigcup_{i=1}^{m+h}(i) A\right)\right)=0 .
$$

So we can choose an open neighbourhood $H$ of zero contained in $H(m+h)$ such that

$$
|\nu|\left\{\left(\bigcup_{y \in S_{0}}\left(y+\bigcup_{i=1}^{m+h}(i) A\right)\right)+H\right\}<\varepsilon
$$

and let $\mathscr{F}$ be the associated positive definite function as in (1). 
We then have, for $\gamma \in \hat{\mathbf{D}_{2}}$,

$$
\begin{aligned}
|\hat{\mu}(\gamma)| & \leqslant \mid\left(\sum_{i=1}^{n} \delta x_{i} * \mu_{i}\right) \hat{(\gamma) \mid+\varepsilon} \\
& \leqslant\left|\int_{\mathbf{D}_{2}} \gamma \mathscr{F} d\left(\sum_{i=1}^{n} \delta x_{i} * \mu_{i}\right)\right|+2 \varepsilon\|\mu\|+\varepsilon \\
& \leqslant\left|\int_{\mathbf{D}_{2}} \gamma \mathscr{F} d\left(\sum_{i=1}^{n} \delta x_{i} * \mu_{i}\right)+\int_{\mathbf{D}_{2}} \gamma \mathscr{F} d \nu\right|+\varepsilon+2 \varepsilon\|\mu\|+\varepsilon(\|\nu\|+2) \\
& \leqslant\left|\int_{\mathbf{D}_{2}} \gamma \mathscr{F} d\left(\sum_{=1}^{n} \delta x_{i} * \mu_{i}+\nu\right)\right|_{+\varepsilon+2 \varepsilon\|\mu\|+\varepsilon(\|\nu\|+2)} \\
& \leqslant\left\|\left(\sum_{i=1}^{n} \delta x_{i} * \mu_{i}+\nu\right) \mid{ }_{\infty} \mathscr{F}(0)+\varepsilon+2 \varepsilon\right\| \mu \|+\varepsilon(\|\nu\|+2)
\end{aligned}
$$

(where the sup norm is taken over $\hat{\mathbf{D}_{2}}$ )

$$
\leqslant(1+\varepsilon)\|(\mu+\nu) \hat{n}\|_{\infty}+(1+\varepsilon)(\varepsilon)+\varepsilon+2 \varepsilon\|\mu\|+\varepsilon(\|\nu\|+2)
$$

and so

$$
\|\hat{\mu}\|_{\infty} \leqslant\|\mu+\nu\|_{\infty}
$$

where the supremum norm is taken over $\hat{\mathbf{D}}$.

From this we have the corollary.

COROllary 1. Let $K \subseteq \mathbf{D}_{2}$ be a compact perfect $K_{2}$ subset of $\mathbf{D}_{2}$ such that $\overline{\mathrm{Gp} K}=\mathbf{D}_{2}$. Then the idempotent associated with the Raikov System generated by $K$ is contained in the closure of the characters $\overline{\mathbf{D}_{2}}$ in $\Delta M\left(\mathbf{D}_{2}\right)$.

Corollary 2. Let $K \subseteq \mathbf{D}_{2}$ be a compact perfect $K_{2}$ subset of $\mathbf{D}_{2}$. Then the idempotent associated with the Raikov System generated by $K$ is contained in the closure of the characters $\overline{\mathbf{D}_{2}}$ in $\Delta M\left(\mathrm{D}_{2}\right)$.

Proof. $\mathbf{D}_{2}=\overline{\mathrm{Gp} K} \oplus H$ for some closed subgroup $H$ of $\mathbf{D}_{2}$. We can give $\mathbf{D}_{2}$ a finer l.c.a. topology $\mathscr{F}$ where

$$
\left(\mathrm{D}_{2}\right)_{\mathscr{F}}=\overline{\mathrm{GpK}} \oplus H_{d}
$$

where $H_{d}$ is the group $H$ with the discrete topology. The positive definite function $F$ with

$$
\begin{array}{lll}
\text { 1. } & F(x)=1 & \forall x \in \overline{\mathrm{Gp} K} \\
\text { 2. } & F(x)=0 & \text { elsewhere }
\end{array}
$$


is continuous on $\left(\mathbf{D}_{2}\right)_{\sigma_{3}}$ and so there exist continuous positive definite functions on $\left(\mathbf{D}_{2}\right)_{\mathscr{G}}$ as required in Theorem 2 . Hence the idempotent $I_{K} \in \overline{\left(\left(\mathbf{D}_{2}\right)_{\mathscr{F}}\right)}$ but $\overline{\left(\left(\mathbf{D}_{2}\right)_{\mathscr{F}}\right)^{\prime}}$ $\subseteq \hat{\mathbf{D}_{2}}$ so $I_{K} \in \hat{\mathbf{D}_{2}}$.

\section{References}

[1] C. Dunkl and D. Ramirez, 'Bounded projections on Fourier-Stieltjes transforms,' Proc. Amer. Math. Soc. 31 (1972), 122-126.

[2] I. Glicksberg and I. Wik, The range of Fourier-Stieltjes transforms of parts of measures (Lecture Notes in Mathematics 266, pp. 73-77, Springer-Verlag (1972)).

[3] E. Hewitt and K. Kakutani, 'A class of multiplicative linear functionals on the measure algebra of a locally compact abelian group,' Illinois J. Math. 4 (1960), 553-574.

\section{Department of Mathematics}

University of New South Wales

Kensington, N.S.W. 2033

Australia 